This article was downloaded by: [National Taiwan University]

On: 28 September 2009

Access details: Access Details: [subscription number 908199084]

Publisher Psychology Press

Informa Ltd Registered in England and Wales Registered Number: 1072954 Registered office: Mortimer House, 37-41 Mortimer Street, London W1T 3JH, UK

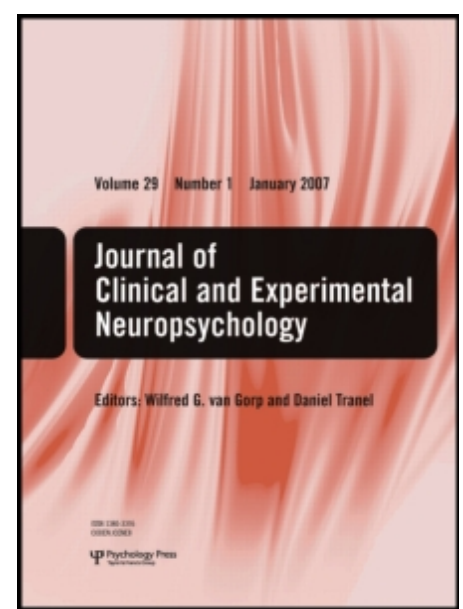

Journal of Clinical and Experimental Neuropsychology

Publication details, including instructions for authors and subscription information:

http://www.informaworld.com/smpp/title content=t713657736

\title{
Neuropsychological Function in Patients with Nasopharyngeal Carcinoma after
}

\section{Radiotherapy}

Mau-Sun Hua; Sien-Tsong Chen; Lok-Ming Tang; Wai-Man Leung

Online Publication Date: 01 October 1998

To cite this Article Hua, Mau-Sun, Chen, Sien-Tsong, Tang, Lok-Ming and Leung, Wai-Man(1998)'Neuropsychological Function in Patients with Nasopharyngeal Carcinoma after Radiotherapy',Journal of Clinical and Experimental Neuropsychology,20:5,684 - 693 To link to this Article: DOI: $10.1076 /$ jcen.20.5.684.1131

URL: http://dx.doi.org/10.1076/jcen.20.5.684.1131

\section{PLEASE SCROLL DOWN FOR ARTICLE}

\footnotetext{
Full terms and conditions of use: http://www.informaworld.com/terms-and-conditions-of-access.pdf

This article may be used for research, teaching and private study purposes. Any substantial or systematic reproduction, re-distribution, re-selling, loan or sub-licensing, systematic supply or distribution in any form to anyone is expressly forbidden.

The publisher does not give any warranty express or implied or make any representation that the contents will be complete or accurate or up to date. The accuracy of any instructions, formulae and drug doses should be independently verified with primary sources. The publisher shall not be liable for any loss, actions, claims, proceedings, demand or costs or damages whatsoever or howsoever caused arising directly or indirectly in connection with or arising out of the use of this material.
} 


\title{
Neuropsychological Function in Patients with Nasopharyngeal Carcinoma after Radiotherapy*
}

\author{
Mau-Sun Hua ${ }^{1}$, Sien-Tsong Chen ${ }^{2}$, Lok-Ming Tang ${ }^{2}$, and Wai-Man Leung ${ }^{3}$ \\ ${ }^{1}$ National Taiwan University, Taipei, Departments of ${ }^{2}$ Neurology, ${ }^{3}$ Radiation Oncology, \\ Chang Gung Memorial Hospital, Taipei, Taiwan
}

\begin{abstract}
A neuropsychological test battery was administered to 27 adult patients with nasopharyngeal carcinoma (NPC) who had received radiotherapy, 28 adult patients with NPC awaiting radiotherapy, and 35 normal adult controls. The 27 patients with radiotherapy had a conventional dosage, $220 \mathrm{cGy}$ per fraction, or less. The median interval between the completion of radiotherapy and the evaluation of neuropsychological function was 1.7 years. Groups were matched for age and educational level. The results showed that our NPC group with radiotherapy had neuropsychological impairments in the areas of auditory attention/concentration, immediate and delayed verbal recall and immediate visual recall, and recent memory, higher-order visuospatial abilities, and bimanual dexterity. Thus neuropsychological dysfunction may occur in patients with NPC within the first 2 years after radiotherapy.
\end{abstract}

Nasopharyngeal carcinoma (NPC) is the most common tumor found in the head and neck among people living in southern mainland China, Hong Kong, and Taiwan. Official surveys recently estimated the incidence of NPC in Taiwan to be 7.37 males and 3.15 females/100,000 population (Cancer Registry Report, 1984).

Radiation therapy is the principal method of treating NPC. Because of the tumor's close proximity to the central nervous system, some of the brain structures are in the track of the radiation beams when patients' basal frontal areas, the olfactory bulbs, the hypothalamus, brain stem, and the spinal cord receive radiation. These brain structures, primarily including the temporal areas and the spinal cord, are often inevitably exposed to considerable dosages of irradiation (Di Lorenzo, Nolletti, \& Palma, 1978; Glass, Hwang, Leavens, \& Libshitz, 1984;
Taso, 1991). Evidence regarding radionecrosis of neural tissue in the central nervous system owing to damage to both nerve fibers and vasculature has been well documented (Lee, 1991; Martins, Johnston, Henry, Stoffel, \& Di Chiro, 1977; Schultheiss, Stephens, \& Maor, 1988) . More specifically, the main sites of lesions include the inferomedial temporal area, cervical spinal cord, pituitary gland, and cranial nerve (Chatani et al., 1986; Lee, Ng, \& Ho, 1988; Woo, Lam, Yu, Lee, \& Huang, 1987).

Extensive evidence confirms the negative effects of radiation therapy on the patient's life expectancy and genetic structures (Roberts \& Bleechman, 1980). However, literature concerning whether radiation therapy has an adverse effect on neuropsychological functioning in patients with NPC who have received only scarce attention.

\footnotetext{
* This paper was presented at the twenty-fifth Annual International Neuropsychological Society Conference, Orlando, Florida, February 6, 1997, and was partly supported by Grant NSC 82-0115-B-182-105 from the National Science Council, Taipei, Taiwan.

Address correspondence to: Mau-Sun Hua, Department of Psychology, National Taiwan University, Taipei 106, Taiwan.E-mail: huams@ccms.ntu.edu.tw.

Accepted for publication: April 24, 1998.
} 
One study (Lee, Hung, Woo, Tai, \& Choi, 1989) has recently explored this issue. The neuropsychological function of a group of 16 adult patients with NPC who had received a complete course of radiotherapy of 2.5 years or more was compared with that of a cohort of 21 patients with NPC who had not yet received radiotherapy. Those results indicated a significantly poor performance on an IQ test that included both verbal and performance intellectual functioning tests, a nonverbal memory recall task, and an immediate verbal recall test in the patients with NPC who received radiotherapy. Meanwhile, those patients also complained of memory loss.

Unfortunately, there are some methodological drawbacks of the study, including the lack of normal controls and use of less than comprehensive neuropsychological function tests. In the current study, we assessed the relevance of neuropsychological examination in monitoring the side effects of radiation treatment in the patients with NPC. In particular, this study addressed the following issues: (1) Is there an impairment of neuropsychological functioning in patients with NPC who have received radiotherapy? and (2) If so, is the defective nature similar to previous observations (Lee et al.,1989)?

\section{METHODS}

\section{Participants}

All participants were adult volunteers. Group 1 included 35 normal healthy adults recruited from the community. Group 2 (NPC-R) consisted of 28 patients who had histologically proven NPC and were awaiting radiotherapy. Group $3(\mathrm{NPC}+\mathrm{R})$ contained 27 patients who had received a complete course of radiation therapy without other types of treatment, such as chemotherapy. Participants in both Groups 1 and 2 were used as controls.

Based on the NPC staging criterion of the International Union Against Cancer (UICC) (Beahrs, Henson, Hutter, \& Kennedy, 1988), only those patients whose tumor proximity did not invade the skull and cranial nerve (i.e., T1, T2, or T3) at initial diagnosis were included in the study (Table 1). The tumor's primary site at initial diagnosis was further confirmed by either computed tomography or magnetic resonance imaging scans. Each patient also received neurological examination and evoked potential studies; those results all revealed normal functioning.

The tumors of the patients in Group 3 were treated with 6-MV photon beam for $4680 \mathrm{cGy} / 26$ fractions $/ 5.5$ weeks through bilateral parallel opposing fields including the entire pharynx, base of the skull, and the neck lymphatics. The fields were then reduced to include the nasopharynx and base of the skull, excluding the spinal cord. By using 10-MV photon from a linear accelerator, all patients but one were treated up to 6480-7280 cGy/38-42 fractions/8-9 weeks. The estimated dose to the temporal lobe was 6480-8400 cGy ( $M$ $=7118 \mathrm{cGy}$ ). In 27 patients (93\%), the basal frontal lobe was definitely included in the radiation field $(M=5226 \mathrm{cGy})$. The neck lymph nodes were boosted with shrinking fields of 9-12 MeV electron beams, up to 2000-3000 cGy.

The mean differences in age and years of education were not statistically significant (Table 1). No participant had a psychiatric history or any apparent emotional problems during the evaluation. All participants were free of dementia, aphasia, and confusional problems, or other significant medical illnesses. All participants, with the exception of 2 patients in Group 2, were right-handed; hand dominance was ascertained according to which hand the participant preferred to use for writing, holding chopsticks, and performing skillful activities.

\section{Tests and Procedure}

Each participant was administered comprehensive neuropsychological test battery at the Neuropsychological Laboratory at the Department of Neurology of Chang Gung Memorial Hospital, Taiwan. The tests, covering intelligence, learning and memory, language and communication, visuospatial reasoning and visuoperception, attention, executive function, and manual dexterity, are listed in Table 2. All of the tests are Chinese versions.

The testing procedure began by recording each participant's demographic data and medical history, followed by tests of attention, intelligence, learning and memory, language and communication, visuospatial and visuoperceptual function, and executive function and manual dexterity. Each participant completed the testing in $4 \mathrm{hr}$. To minimize the effects of fatigue on performance, all participants completed the testing in two sessions (2 $\mathrm{hr}$ between sessions).

\section{Data Analysis}

One-way multivariate analysis of variance (MANOVA) was used to examine whether a performance difference arose among the three groups on the neuropsychological tests. Significant 
Table 1. Demographic Data.

Note . NPC-R = Nasopharyngeal cancer without radiotherapy; NPC+R = Nasopharyngeal cancer with radiotherapy; 1 = Tumor limited to one subsite of nasopharynx; T2 = Tumor invades more than one subsite of nasopharynx; T3 = Tumor invades nasal cavity and/or oropharynx.

${ }^{a}$ Beahrs et al. (1988) staging criteria. 
Table 2. Neuropsychological Function Tests.

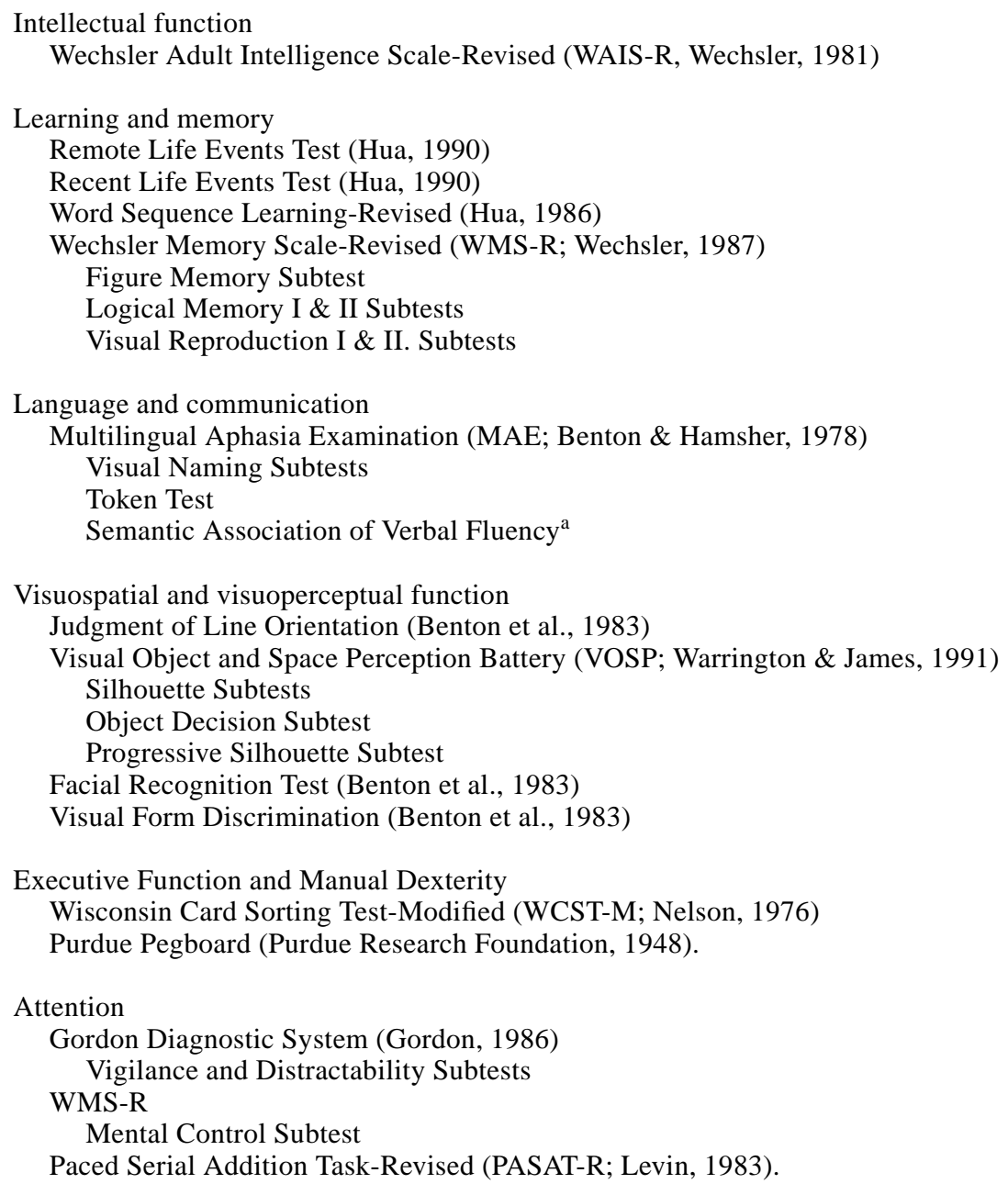

${ }^{a}$ Used to measure associative verbal fluency for animal, fruit and vegetable, and replace the original subtest of Controlled Oral Word Association of the MAE.

MANOVA effects were subsequently analyzed by using analysis of variance, when appropriate, with post hoc pairwise contrasts. Bonferroni corrections were used to control for Type I error.

\section{RESULTS}

Table 3 shows the mean scores and information regarding statistical testing for each dependent measure for the three groups. On the intellectual function assessment (WAIS-R), the performance of normal controls was best, followed by NPC patients who were waiting for radiotherapy, and then by NPC patients who had received radiation therapy on all the WAIS-R. However, the score difference among the three groups was not statistically significant.

On the semantic memory function tests (i.e., both the Remote and Recent Life Events Tests), NPC group with radiotherapy again performed poorer than the normal controls and the NPC 
group without radiation treatment; meanwhile, the performance of the NPC patients without radiotherapy was compatible with that of the normal control group. The difference among the three groups, however, only appeared on the Recent Life Events. Further analysis with the Scheffé's pairwise comparison indicated a statistically significant difference between the normal controls and the patients with NPC who have received radiotherapy. On the episodic memory tests (i.e., Word Sequence Learning, and five subtests of WMS-R), the performance of the normal controls was superior to that of the patients with or without radiotherapy with the exception of Logical Memory I and II, and Visual Reproduction II. The difference among the three groups, however, only arose on the Word Sequence Learning and Figure Memory. The Scheffé' post hoc pairwise comparison was further used to examine the performance difference on these tests among the groups. For Word Sequence Learning, the differences of both correct scores and a 10-min delayed recall score between the normal controls and the patients with NPC who had received radiotherapy reached a statistically significant level. On the Figure Memory subtest of the WMS-R, only the difference between the normal controls and the patients with radiotherapy was statistically significant.

On the language and communication functioning tests, the performance of the patients with radiotherapy was compatible to that of the normal controls, and that of the patients without radiotherapy. Data anaylysis indicated that the score difference between the normal controls and the NPC patients was not statistically significant.

Regarding visuospatial and visuoperceptual functioning, normal controls performed better than the patients with NPC. However, the difference (which reached a statistically significant level among the three groups) was only noted on the Silhouette and the Object Decision of the VOSP. Post hoc pairwise comparisons with Scheffé's procedure indicated that significant differences were evident between normal controls and both groups of the patients with NPC on these two subtests.
For the executive function tests, the patients with NPC who had received radiotherapy performed worse than the normal controls and the patients with NPC who had not yet received radiation therapy. However, the score differences were not statistically significant. With regard to manual dexterity, the patients with radiotherapy performed worse than did the normal controls and the patients without radiation therapy on the Purdue Pegboard. The score difference was statistically significant among the three groups. Further data analysis indicated that the patients without radiotherapy performed significantly better than did the patients with radiotherapy on the three tasks of the test.

On the attention tasks, the patients with radiotherapy performed poorer than did the normal controls and the patients without radiotherapy. However, no significant difference was evident among the three groups with the exception of the Mental Control Subtest of the WMS-R: The patients with radiotherapy performed worse than did the normal controls and the patients without radiotherapy on the Mental Control Test. However, only the score difference between the normal controls and the patients with radiation was statistically significant.

\section{DISCUSSION}

Our patients with NPC displayed some neuropsychological function changes after radiotherapy as compared to their normal counterparts. The impairments included auditory attention/concentration, learning and memory, and high-level visuospatial reasoning.

As the subtest of the Mental Control of the WMS-R primarily measures auditory focused attention/concentration (Roth, Conboy, Reeder, $\&$ Boll, 1990), the poor performance on the test noted in the patients with NPC who had received radiotherapy reflected their difficulty in this type of cognitive function. The patients, however, did not have any remarkable difficulty in performing other visual attention tests (i.e., the Vigilance and Distrability subtests of the Gordon Diagnostic System), and an auditory sustained attention task PASAT-R (Gronwall, 
Table 3. Comparisons of Neuropsychological Test Results.

\begin{tabular}{|c|c|c|c|c|c|c|c|c|}
\hline & \multicolumn{2}{|c|}{ Controls $(n=35)$} & \multicolumn{2}{|c|}{ NPC-R $(n=28)$} & \multicolumn{2}{|c|}{$\mathrm{NPC}+\mathrm{R} \quad(n=27)$} & \multirow{2}{*}{$\begin{array}{c}F \\
\text { (ANOVA) }\end{array}$} & \multirow[b]{2}{*}{$P$} \\
\hline & $M$ & $(S D)$ & $M$ & $(S D)$ & $M$ & $(S D)$ & & \\
\hline \multicolumn{9}{|l|}{ Intellectual Function } \\
\hline VIQ & 100.91 & $(15.38)$ & 99.11 & $(16.24)$ & 93.56 & $(14.58)$ & 1.82 & 0.17 \\
\hline PIQ & 94.12 & $(12.50)$ & 90.64 & $(12.98)$ & 88.48 & $(12.34)$ & 1.85 & 0.21 \\
\hline FSIQ & 97.32 & (14.19) & 95.36 & $(14.94)$ & 90.41 & $(13.56)$ & 1.94 & 0.16 \\
\hline \multicolumn{9}{|l|}{ Memory Function } \\
\hline Remote Life Events Test Recall & 47.80 & $(2.46)$ & 46.96 & $(3.29)$ & 46.30 & $(3.33)$ & 1.94 & 0.15 \\
\hline Recent Life Events Test Recall & 38.94 & (1.94) & 37.89 & $(3.11)$ & 36.81 & $(3.30)$ & 4.50 & $0.01 *$ \\
\hline \multicolumn{9}{|l|}{ Word Sequence Learning } \\
\hline Correct & 53.89 & $(5.02)$ & 51.14 & $(8.68)$ & 48.67 & $(9.38)$ & 3.52 & $0.03 *$ \\
\hline Recall & 4.00 & $(1.41)$ & 3.11 & $(1.71)$ & 2.96 & $(1.72)$ & 3.92 & $0.02 *$ \\
\hline \multicolumn{9}{|l|}{ WMS-R } \\
\hline Logical Memory I & 19.13 & $(7.76)$ & 23.75 & $(10.72)$ & 19.25 & $(9.69)$ & 2.17 & 0.12 \\
\hline Logical Memory II & 15.74 & (7.61) & 19.89 & $(11.70)$ & 16.67 & $(10.76)$ & 1.42 & 0.25 \\
\hline Figure Memory & 7.23 & $(1.44)$ & 6.93 & $(1.21)$ & 6.33 & $(1.59)$ & 3.06 & $0.05 *$ \\
\hline Visual Reproduction I & 37.17 & $(5.57)$ & 35.54 & $(6.24)$ & 33.96 & $(6.40)$ & 2.17 & 0.12 \\
\hline Visual Reproduction II & 31.94 & $(9.35)$ & 32.00 & $(7.95)$ & 31.44 & $(9.46)$ & 0.03 & 0.97 \\
\hline \multicolumn{9}{|l|}{ Language Function } \\
\hline Visual Naming & 54.74 & $(5.76)$ & 54.86 & $(5.40)$ & 54.00 & (4.99) & 0.21 & 0.81 \\
\hline Semantic Association of Verbal Fluency & 36.47 & $(9.69)$ & 37.96 & $(7.91)$ & 37.48 & $(8.86)$ & 0.23 & 0.80 \\
\hline Token Test & 42.02 & $(2.36)$ & 42.29 & $(1.72)$ & 41.48 & $(3.09)$ & 0.78 & 0.46 \\
\hline \multicolumn{9}{|l|}{ Visuopatial Function } \\
\hline Judgement of Line Orientation & 23.54 & $(4.71)$ & 22.75 & $(4.19)$ & 23.33 & $(3.43)$ & 0.29 & 0.75 \\
\hline \multicolumn{9}{|l|}{ VOSP } \\
\hline Silhouette & 18.23 & $(4.31)$ & 15.43 & $(4.46)$ & 14.74 & $(3.83)$ & 5.58 & $0.00 *$ \\
\hline Object Decision & 16.20 & $(3.56)$ & 13.79 & (3.04) & 13.74 & $(3.34)$ & 5.22 & $0.00 *$ \\
\hline Progressive Silhouette & 11.32 & $(2.81)$ & 12.32 & $(2.78)$ & 12.45 & $(2.60)$ & 1.54 & 0.25 \\
\hline \multicolumn{9}{|l|}{ Visuoperception Function } \\
\hline Facial Recognition Test & 43.54 & $(5.57)$ & 44.12 & $(3.22)$ & 43.37 & $(4.41)$ & 0.19 & 0.83 \\
\hline \multirow[t]{2}{*}{ Visual Form Discrimination } & 29.60 & $(2.38)$ & 27.77 & $(3.66)$ & 27.96 & $(4.00)$ & 2.88 & 0.06 \\
\hline & & & & & & & \multicolumn{2}{|c|}{ (Table continues } \\
\hline
\end{tabular}




\begin{tabular}{|c|c|c|c|c|c|c|c|c|}
\hline & \multicolumn{2}{|c|}{ Controls $(n=35)$} & \multicolumn{2}{|c|}{ NPC-R $(n=28)$} & \multicolumn{2}{|c|}{$\mathrm{NPC}+\mathrm{R} \quad(n=27)$} & \multirow{2}{*}{$\begin{array}{c}F \\
\text { (ANOVA) }\end{array}$} & \multirow[b]{2}{*}{$p$} \\
\hline & $M$ & $(S D)$ & $M$ & $(S D)$ & $M$ & $(S D)$ & & \\
\hline \multicolumn{9}{|l|}{ Executive Functioning } \\
\hline \multicolumn{9}{|l|}{ WCST-Modified } \\
\hline number complete categories & 5.42 & $(1.88)$ & 5.39 & $(1.75)$ & 5.07 & $(2.15)$ & 0.29 & 0.75 \\
\hline number of perseverative errors & 2.74 & $(7.13)$ & 2.21 & $(3.15)$ & 2.77 & $(3.01)$ & 0.11 & 0.90 \\
\hline \multicolumn{9}{|l|}{ Manual Dexterity } \\
\hline \multicolumn{9}{|l|}{ Purdue Pegboard } \\
\hline dominant hand & 14.64 & $(2.30)$ & 15.81 & $(1.89)$ & 14.46 & $(1.89)$ & 3.60 & $0.03 *$ \\
\hline non-dominant hand & 13.82 & $(2.62)$ & 14.63 & $(1.78)$ & 13.09 & $(1.69)$ & 3.64 & $0.03 *$ \\
\hline both hands & 12.27 & $(1.90)$ & 12.75 & (1.78) & 11.36 & $(1.90)$ & 3.97 & $0.02 *$ \\
\hline \multicolumn{9}{|l|}{ Attention } \\
\hline \multicolumn{9}{|l|}{ Vigilance Task } \\
\hline correct & 28.14 & $(2.12)$ & 27.25 & $(4.69)$ & 26.63 & $(3.28)$ & 1.52 & 0.22 \\
\hline commission & 3.48 & $(5.51)$ & 2.86 & (1.98) & 2.78 & $(2.55)$ & 0.32 & 0.73 \\
\hline \multicolumn{9}{|l|}{ Distractability Task } \\
\hline correct & 28.60 & (1.94) & 28.29 & $(1.67)$ & 28.26 & $(2.35)$ & 0.29 & 0.75 \\
\hline commission & 3.48 & $(4.65)$ & 2.36 & $(1.59)$ & 2.44 & $(2.44)$ & 0.18 & 0.83 \\
\hline PASAT-R & 72.63 & $(17.03)$ & 72.89 & $(18.15)$ & 66.81 & (18.39) & 1.05 & 0.36 \\
\hline Mental Control & 5.06 & $(1.08)$ & 4.68 & $(1.28)$ & 3.96 & $(1.53)$ & 5.53 & $0.00 *$ \\
\hline
\end{tabular}

Note. For the Recent Life Events Test, Word Sequence Learning Test (correct and recall), Figural Memory Test and Mental Control Test, Controls differed significantly from NPC+R $(p<.02$ for Recent Life Events Test, $p<.04$ for Word Sequence Learning Test-correct, $p<.03$ for Word Sequence Learning Test-recall, $p=.05$ for Figure Memory, and $p<.01$ for Mental Control); For the Silhouette and Object Decision Subtests, Controls differed from NPC-R, and Controls differed from NPC+R ( $p<.006$ for Silhouette Subtest, $p<.008$ for Object Decision Subtest); For the Purdue Pegboard Test (dominant hand, non-dominant hand and both hands), NPC-R differed significantly from NPC+R ( $p<.04$ for dominant hand and non-dominant hand, and $p<.03$ for both hands).

* Statistically significant 
1987). Thus, our patients with radiotherapy apparently had difficulty in modality-specific (i.e., auditory) and an aspect-specific (i.e., focused) attention, rather than in general attention. Could this deficit be attributed to the effects of medication, or the patients' fatigue or emotional disturbances, such as anxiety and depression during the testing? The answer might not be fully clear because, in other instances, modality- and aspect-related attention functions were preserved in these patients.

With respect to learning and memory, our patients with radiotherapy manifested problems in both verbal and visual memory functions. The impairment of verbal memory included both semantic and episodic types were reflected primarily by the Recent Life Events Test and Word Sequence Learning, respectively. The defect of visual memory mainly involved the immediate dimension that was essentially expressed by poor performance on the subtest of Figure Memory of the WMS-R (Roth, Conboy et al., 1990). However, those patients did not have any difficulty in performing the Visual Reproduction I Subtest. Both the Figure Memory and the Visual Reproduction I are conventionally used to measure immediate visual memory functions; the nature of test items for both tests are similar: that is, geometric figures. Normal performance on these two tests not only demands immediate memory, but also visuoperception of geometric figures, with the exception of an additional segment of visual motor skills in performing the Visual Reproduction I. Such contradictory results in this study are difficult to clarify. A possible explanation for this divergent outcome is that the items in the Visual Reproduction I subtest are more accessible than are those in the Figure Memory subtest for an individual to conceptualize verbally (Lezak, 1995). Thus, the fact that our patients could perform well normally on the Visual Reproduction I subtest may be due to their use of a verbal mnemonic strategy.

Spatial abilities consist of two processes in humans, the lower-level and the higher-level (Levin et al., 1991; Ogden, 1990). According to Ogden's proposal, the lower-level visual-spatial processes primarily involve the perception of shape difference and line orientation, whereas the higher-level visual-spatial processes associate the abstraction of physical stimuli, mental rotation, and the mental transformation of shapes. In the same manner, the Silhouette and Object Decision subtests of the VOSP not only measure the lower-level visuospatial and visuoperceptual process, but also the higherlevel visuospatial process and the function of verbal identification of real objects. Our patients with radiotherapy encountered difficulties in solving both the Silhouette and Object Decision tasks in the context of normal performance on the Judgement of Line Orientation, Facial Recognition, and Visual Form Discrimination tasks, as well as verbal confrontation naming. Thus, the patients were apparently impaired in the higher-level visuospatial process.

Compared to the patients awaiting radiotherapy, the patients with radiation therapy only showed impairment of manual dexterity of both hands expressed primarily the Purdue Pegboard. This result regarding manual dexterity contradicts the earlier observations by Lee and his colleaugues (1989) who reported poor intellectual and immediate verbal and delayed nonverbal memory functionings in their patients with NPC who received radiotherapy as compared with their counterparts of the patients with NPC who were awaiting radiation treatment. Apparently, the neuropsychological deficits manifested in the patients with NPC, who had received radiotherapy in the study of Lee et al. were more extensive than those in the current study. In addition, the probable brain damage reflected by the neuropsychological impairment implicates a wider involvement in the patients with radiotherapy in the Lee et al. study than in the current study. Two factors might account for such a discrepancy. One may be that the patients with NPC in the study of Lee and his colleagues received a higher dose of radiation ( $350 \mathrm{cGy}$ per fraction on average ) than did our patients (180-220 cGy per fraction). Thus, their patients potentially had a more extensive impact on brain functions. Another factor may be related to the fact that duration from completion of radiotherapy to neuropsychological examination is different in the two studies. The interval between the completion of radiation treatment and the neuro- 
psychological function evaluation was longer in the Lee et al. study ( at least 2.5 years after radiotherapy for each of the patients, with a median of 5.5 years ranging from 2.5-10.2 years) than in the current study (a median of 1.7 years ranging from 7 days -9 years). What is unknown is whether extensive brain dysfunction only occurred at some certain length of time after radiotherapy, such as a median of 5 years after the treatment (as the patients in the Lee et al. study) and, consequently, the widely diverse effects on neuropsychological funtioning were manifested in these patients. In fact, radionecrosis of brain structures, such as the temporal lobes, has been observed in the patients who suffered NPC with an average interval of 5 years following radiotherapy (Woo et al., 1987). Further investigation on this issue is necessary. Nevertheless, our findings might imply that less extensive brain dysfunction resulting from radiotherapy could occur two years after the treatment.

Compared with the normal control subjects, the patients with NPC who were awaiting radiotherapy unexpectedly exhibited defects in higher-order visuospatial functioning on the Silhouette and Object Decision subtests of the VOSP. These results were quite unexpected and confusing. Because the patients were not exposed to radiation, brain dysfunction due to radiotherapy could not account for these deficits. Also it is unknown whether psychological distress regarding the recently discovered physical illness, as Lee et al. (1989) suggested, could adversely affect patient's performance on these tasks.

Overall, our patients with NPC who have received radiation treatment manifested deficits in auditory focused attention, both verbal and visual memory, and manual dexterity as compared to both control groups. This extensive impairment of psychological functions suggested dysfunction of the bilateral frontal, mesial temporal, or frontotemporal lobes.

Regarding visuospatial function, difficulties encountered in the higher-level visuospatial process in our patients with radiotherapy might indicate an adverse effect of radiation treatment on the frontal, parietal, and inferotemporal structures. However, such an explanation cannot be applied to patients without radiation treatment who also experienced difficulties in the higher-level visuospatial process. Whether impairment of the higher-level visuospatial abilities in the patients with and without radiotherapy could result from two independent pathological origins (neurological and psychological) remains unclear and warrants further investigation.

In summary, our study indicates that the patients with NPC who have been treated with radiation therapy definitely show neuropsychological deficits including auditory focused attention/ concentration, immediate and delayed verbal recall and immediate visual recall, recent memory, and manual dexterity. If these results are replicable, adequate tests to examine the neuropsychological functioning of the patient with NPC may be useful in detecting the potential side effects of radiation therapy. Such an avenue would encourage radiation oncologists to proceed with radiotherapy but impose as minimal adverse effects on these patients as possible.

\section{REFERENCES}

Beahrs, O.H., Henson, D.E., Hutter, R.V.P., \& Kennedy, B.J. (Ed.) (1988). Manual for staging of cancer (4th ed.) (pp. 34-35). Philadelphia: J.B. Lippincott.

Benton, A.L., \& Hamsher, K. deS. (1978). Multilingual Aphasia Examination. Iowa City, IA: University Hospital of Iowa, Department of Neurology.

Benton, A.L., Hamsher, K. deS., Varney, N. R., \& Spreen, O. (1983). Contributions to neuropsychological assessment: A clinical manual. NY: Oxford University Press.

Cancer Registry Report (1984). Taiwan. Taiwan, ROC: Department of Health, The Executive Yuan.

Chatani, M., Testima, T., Inoue, T., Azuma, I., Yoshimura, H., Oshitani, T., Hashiba, M., Nishiyama, K., Tsutsui, K., \& Fujimura, T.(1986). Radiation therapy for nasopharyngeal carcinoma: Retrospective review of 105 patients based on a survey of Kansai Therapist Group. Cancer, 57, 2267-2271.

Di Lorenzo, N., \& Nolletti, A., \& Palma, L. (1978). Late cerebral radionecrosis. Surgical Neurology, 10, 281-290. 
Glass, J. P., Hwang, T-L, Leavens, M. E., \& Libshitz, H. I. (1984). Cerebral radiation necrosis following treatment of extracranial malignancies. Cancer, 54, 1966-1972.

Gordon, M. (1986). The Gordon Diagnostic System. DeWitt, NY: Gordon System.

Gronwall, D. (1987). Advances in the assessment of attention and information processing after head injury. In H.S. Levin \& H.M. Eisenberg (Eds.), Neurobehavioral recovery from head injury (pp.355-371). NY: Oxford University Press.

Hua, M.-S.(1986). Word sequence learning-Revised. Unpublished manuscript. Chungli, Taiwan: Chung Yuan University, Department of Psychology.

Hua, M.-S.(1988). Divided and focused tasks. Unpublished manuscript. Chungli, Taiwan: Chung Yuan University, Deparment of Psychology.

Hua, M.-S.(1990). Remote and recent life events tests. Unpublished manuscript. Chungli, Taiwan: Chang Yuan University, Department of Psychology.

Lee, A.W.H., Ng, S.H., \& Ho, J.H.C. (1988). Clinical diagnosis of lateral temporal lobe necrosis following radiation therapy for nasopharyngeal carcinoma. Cancer , 61, 1535-1542.

Lee, A.W.M. (1991). Complications of radiotherapy. In C.A.V. Hasselt, \& A.G. Gibb (Eds.), Nasopharyngeal carcinoma. (pp. 221-234). Hong Kong: The Chinese University Press.

Lee, P.W., Hung, B.K.M., Woo, E.K.W., Tai, P.T.H., \& Choi, D.T.K. (1989). Effects of radiation therapy on neuropsychological functioning in patients with nasopharyngeal carcinoma. Journal of Neurology, Neurosurgery, \& Psychiatry, 52, 488-492.

Levin, B.E., Elabre, M.M., Reisman, S., Weiner, W.J., Sanchez-Ramos, V., Singer, C., \& Brown, M.C. (1991). Visouspatial impairment in Parkinson's disease. Neurology, 41, 365-369.

Levin, H.S. (1983). Paced Auditory Serial Addition Task-revised. Unpublished manuscript. Galveston, TX: University of Texas Medical Branch at Galveston, Neurosurgery Division.

Lezak, M.D. (1995). Neuropsychological assessment (3rd. ed.). NY: Oxford University Press.
Martins, A.N., Johnston, J.S., Henry, J.M., Stoffel, T.J., \& Di Chiro. (1997) Delayed radiation necrosis of the brain. Journal of Neurosurgery , 47, 336345.

Nelson, H.E. (1976). A modified card sorting test sensitive to frontal lobe defects. Cortex, 12, 313-324.

Ogden, J.A. (1990). Spatial abilities and deficits in aging and age-related disorders. In F. Boller \& J. Grafman (Eds.), Handbooks of neuropsychology. (Vol. 4, pp. 265-278). Amsterdam : Elsevier.

Purdue Research Foundation. (1948). Examiner's manual for the Purdue Pegboard. Chicago: Science Research Associates.

Roberts, T., \& Bleecham, N.M. (1980). Health hazards of radiotherapy. Public Health Review, 9, 3766.

Roth, D.L., Conboy, T.S., Reeder, K.P., \& Boll, T.J. (1990). Confirmatory factor analysis of the Wechsler Memory Scale-Revised in a sample of headinjured patients. Journal of Clinical and Experimental Neuropsychology, 12, 834-842.

Schultheiss, T.E., Stephens, L.C., \& Maor, M.H. (1988). Analysis of the histopathology of radiation myelopathy. International Journal of Radiation Oncology, Biology, \& Physics, 14, 27-52.

Taso, S.Y. (1991). Radiotherapy. In C.A. van Hasselt \& A.G. Gibb (Eds.), Nasopharyngeal carcinoma. Hong Kong: The Chinese University Press.

Warrington, E.K., \& James, M. (1991). Visual object spatial perception. Suffolk, UK : Thames Valley Test Company.

Wechsler, D. (1981). Wechsler Adult Intelligence Scale-Revised manual. San Antonio, TX: Psychological Corp.

Wechsler, D. (1987). Wechsler Memory Scale-Revised manual. San Antonio, TX: Psychological Corp.

Woo, E., Lam, K., Yu, Y.L., Lee, P.W.H., \& Huang, C.Y. (1987). Cerebral radionecrosis: Is surgery necessary? Journal of Neurology, Neurosurgery, \& Psychiatry, 50, 1407-1414. 